\author{
Dhruv Patel ${ }^{1}$, Monika \\ Zwierzchoniewska ${ }^{1}$ and Bulang $\mathrm{He}^{1,2 *}$ \\ ${ }^{1}$ Liver and Kidney Transplant Unit, Sir Charles \\ Gairdner Hospital, Australia \\ ${ }^{2}$ School of Surgery, The University of Western \\ Australia, Australia \\ Dates: Received: 15 March, 2015; Accepted: 24 \\ April, 2015; Published: 27 April, 2015 \\ *Corresponding author: Bulang $\mathrm{He}$, School of \\ Surgery, The University of Western Australia, \\ Australia, E-mail: bulang.he@health.wa.gov.au \\ www.peertechz.com \\ ISSN: 2454-2968
}

\section{Research Article \\ Dual Kidney Transplant: Clinical Experience and Overview of Surgical Techniques}

\begin{abstract}
Background: Dual Kidney Transplant (DKT) of marginal kidneys has expanded utilizing the extended criteria donor (ECD) organs. The aim of this study is to report the outcomes of dual kidney transplant in our institute and review the surgical techniques of DKT in the Literature.

Materials and Methods: Between Jan 1999 and April 2014, 10 cases (8 male and 2 female mean age 50 years) of DKT were performed in our institute. The surgical techniques include unilateral incision for 9 patients, a midline incision for 1 patient. The literature was reviewed for surgical techniques of DKT.

Results: Two recipients had DKT as an En bloc from pediatric donors, whereas others had DKT from elderly donors. The mean surgical time was 5 hours 4 min (Range: 3 hours 40 min to 6 hours 24 $\mathrm{min})$. Two patients had a complication of lymphocele and subsequent development of ureteric stricture. There was no vessel thrombosis and no urine leakage in this cohort. The creatinine level was 137 ( \pm 37.4) $\mu \mathrm{mol} / \mathrm{L}$ at 12 months follow-up. The various techniques were described in the literature.

Conclusion: DKT is an established technique for expanding use of extended criteria of kidney donors. The clinical outcome is satisfactory and surgical complications can be minimized. Based on our experience in a small series, vascular complication can be prevented with caution during vesse anastomosis and position of the kidney grafts. The single site placement of DKT is preferred from the literature review.
\end{abstract}

\section{Introduction}

It has been well recognized that kidney transplant is the best modality for treatment of end stage kidney disease. The shortage of donors and continuous growing of waiting list for kidney transplantation is an ongoing problem that the transplant community has faced for many years. Dual kidney transplantation (DKT) of marginal kidneys to a single recipient, allows utilization of ECDs to alleviate the disparity between available donors and potential recipients. Many centers now perform DKT using different surgical techniques, including the bilateral placement of both kidneys, in the intra- or extra-peritoneal cavity, through two separate Gibson incisions or one midline incision, and the unilateral placement of both kidneys in the extra peritoneal cavity through a single Gibson incision. The aim of this study is to report the surgical techniques and outcomes of our small series of DKT. The surgical techniques for DKT were also reviewed from the literature.

\section{Materials and Methods}

The study was approved by human research ethic committee of the institute. From January 1999 to April 2014, 10 cases of DKT were performed in our institute. The medical records were retrospectively reviewed and the data of surgical technique, perioperative variables surgical complications, patients and graft outcomes were extracted. The theatre electronic database was also searched for the record of surgical time. The wedge biopsy was performed immediately following kidney procurement and the histopathology results were taken into consideration as one selection criterion for decision-making on DKT. The patients were contacted by mail for self-measurement of the wound length. All parameters were recorded as Mean \pm SD.

State selection criteria for DKT: DKT is considered if the donor meets 3 or more of 6 criteria listed below.

1) Donor age $>70$ years

2) Best eGFR $<60 \mathrm{ml} / \mathrm{min}$ or persistently high serum creatinine

3) Evidence of severe vascular disease (confirmed at organ procurement)

4) Diabetes or Hypertension,

5) 20 to $40 \%$ Glomerulosclerosis on biopsy

6) Proteinuria $(\mathrm{ACR}>30, \mathrm{PCR}>50)$. (ACR: albumin creatinine ratio; PCR: protein creatinine ratio)

Delayed graft function (DGF) is defined as the patient required dialysis in the first week after kidney transplant [1].

\section{Operative techniquesOrgan retrieval and preparation}

Dual kidneys from adult deceased donors (DDs) were retrieved in the usual method, separated at the time of retrieval, flushed and preserved in cold University of Wisconsin (UW) solution for transportation to recipient hospital. Then, both kidneys were prepared separately on back table as a normal procedure. However, dual kidneys from pediatric donors $(<15 \mathrm{~kg})$ were retrieved as an 
En Bloc along with the distal donor aorta and inferior vena cava for transplantation. Care was taken during the organ preparation to ligate the branches from vena cava and aorta. The superior end of aorta and vena cava were closed with running suture of 5-0 Prolene. All the donor kidneys except of small paediatric donors used for DKT were biopsied preoperatively.

\section{Dual kidney transplantation}

The kidneys were transplanted either unilaterally, bilaterally or en bloc using classic Gibson incision for Unilateral DKT (UDKT) $(\mathrm{n}=7)$ and En-bloc DKT (EDKT) $(\mathrm{n}=2)$ or midline incision for Bilateral DKT (BDKT) $(\mathrm{n}=1)$. Right iliac fossa was the preferred side for UDKT and only one UDKT and one EDKT were performed in the left iliac fossa. Both Kidneys were placed at extra-peritoneal space in the iliac fossa. The renal artery was anastomosed to external iliac artery in end-to-side fashion. The renal vein was anastomosed in endto-side fashion to the external iliac vein. In UDKT, left kidney was placed superiorly in five cases where as right kidney was superior in two cases. One patient was for his third time of kidney transplant. The dual grafts were placed at right iliac fossa, where the previous kidney graft was removed prior to being listed. In En-bloc DKT, the inferior end of vena cava and aorta was anastomosed to side of external iliac vein and external iliac artery respectively. The ureter was anastomosed to the bladder separately in 3 cases whereas the two ureters were joined together as one anastomosis (Lich-Gregoir technique [2] to the bladder in 7 cases. The ureteric stent was inserted in the ureter as routine practice that is the same as in conventional single kidney transplant in our institute.

\section{Results}

The transplant surgery was success in all 10 recipients (Table 1). The mean surgical time was 304 minutes, ranged from 220 to 384 minutes. The mean vessel anastomotic time was 48 minutes for the first kidney and 65 minutes for second kidney graft. The vessel anastomotic time was 59 minutes for En bloc kidney transplant. The estimated blood loss was minimal in most cases except in two cases where $500 \mathrm{ml}$ and $1800 \mathrm{ml}$ blood loss was recorded. The cold ischemic time was from 8.5 hours to 16 hours (average 12.3 hours). Two patients developed delayed graft function (DGF). One patient was required Hemodialysis (HD) twice on post-operative day 0 and day 3 respectively; Whereas another patient was required continuous veno-venous Haemofiltration (CVVH) for 24 hours immediately post surgery due to hyperkalemia and required another 12 hours of CVVH on day 3 due to DGF. Of ten patients, two had a symptomatic lymphocele formation post kidney transplant. The lymphocele was treated by laparoscopic fenestration and resolved completely. One of them who had a midline incision with bilateral placement of kidney graft subsequently developed a ureteric stenosis on left side; while another patient with unilateral kidney placement developed dual ureteric stenosis. The surgical urinary tract reconstruction was performed in both cases. There was no further complication. Seven of 10 recipients returned the form of incision measurement with average length in $20 \mathrm{~cm}(10 \mathrm{~cm}-29 \mathrm{~cm})$.

There was no surgical graft loss, vessel thrombosis or urine leakage in this series. The mean length of hospital stay was 9 days ( 5 to
17 days). The mean serum creatinine (SCr) level was $137 \pm 38 \mu \mathrm{mol} / \mathrm{L}$ at 12 months follow-up (Figure 1a). The mean estimated glomerular filtration rate (eGFR) was $49 \mathrm{~mL} / \mathrm{min} / 1.73 \mathrm{~m}^{2}$ at 12 months follow up (Figure 1b).

One recipient developed dual kidney grafts failure10 years after transplant due to chronic rejection. He subsequently received a second kidney transplant at the left iliac fossa. All patients had minimum 1 year follow up.

\section{Discussion}

To overcome the disparity between the organ donation and demand of the kidney transplantation, various strategies have been proposed including use of kidneys from ECD [3]. DKT was first reported in 1996 [4] and has been widely accepted ever since by many centres to meet the increasing demand of kidney transplantation [57]. Concerns were raised regarding inadequate nephron mass and expected life span of the ECD kidneys. However, literature review reveals satisfactory outcome of DKT in terms of graft function [8].

In our experience, we have found that DKT is a better modality for utilisation of marginal kidneys as it retains maximal nephron

\begin{tabular}{|c|c|c|c|c|}
\hline & EDKT $(n=2)$ & UDKT $(n=7)$ & BDKT $(n=1)$ & Total \\
\hline \multicolumn{5}{|c|}{ Side of Implantation (n) } \\
\hline Right & 1 & 7 & - & 8 \\
\hline Left & 1 & 0 & - & 1 \\
\hline $\begin{array}{l}\text { Length of Incision } \\
(\text { mean } \mathrm{cm})\end{array}$ & $\mathrm{n} / \mathrm{a}$ & 20 & 10 & $\mathrm{n} / \mathrm{a}$ \\
\hline Surgical Time (min) & $230 \pm 13$ & $314 \pm 51$ & 384 & $303 \pm 63$ \\
\hline \multicolumn{5}{|c|}{ Cold Ischaemia Time (min) } \\
\hline First Kidney & \multirow{2}{*}{$737 \pm 315$} & $643 \pm 275$ & $\mathrm{n} / \mathrm{a}$ & $690 \pm 256$ \\
\hline Second Kidney & & $708 \pm 274$ & $\mathrm{n} / \mathrm{a}$ & $722 \pm 255$ \\
\hline \multicolumn{5}{|c|}{ Warm Ischaemia Time (min) } \\
\hline First Kidney & \multirow{2}{*}{$59 \pm 8$} & $48 \pm 8$ & $\mathrm{n} / \mathrm{a}$ & $53 \pm 9$ \\
\hline Second Kidney & & $65 \pm 32$ & $\mathrm{n} / \mathrm{a}$ & $62 \pm 27$ \\
\hline
\end{tabular}

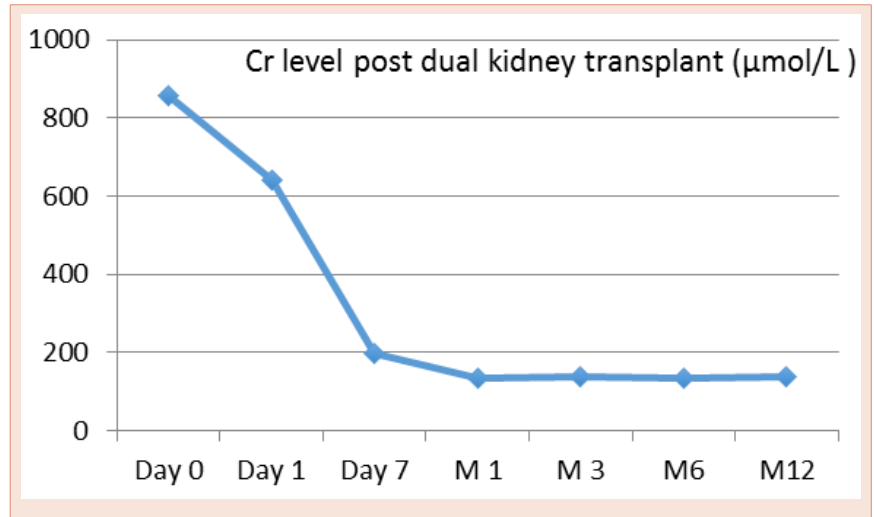

Figure 1a: The mean $\mathrm{Cr}$ level post dual kidney transplant over 12 months follow up. 


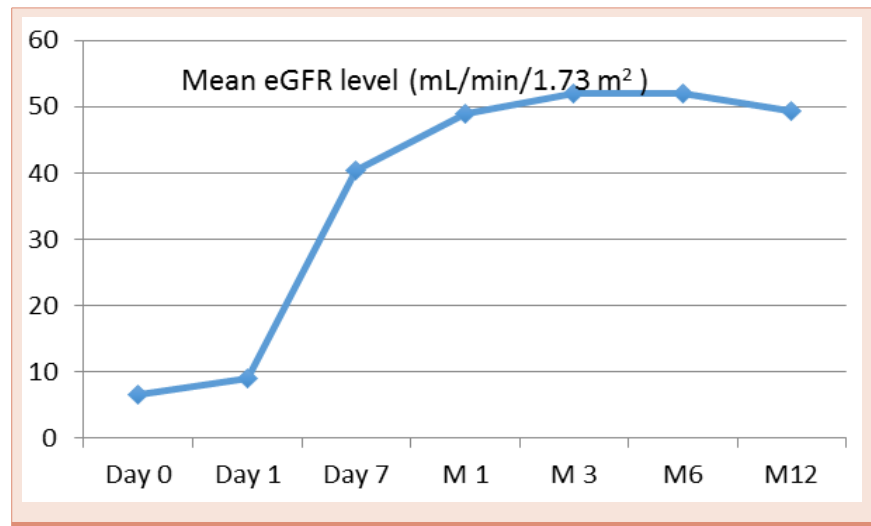

Figure 1b: The mean eGFR post dual kidney transplant over 12 months follow up.

mass and hence graft function. In this small cohort, all patients have satisfactory kidney function and live a good quality of life as any other recipients who received a single kidney transplant from standard criteria donor.

In this series, the graft survival rate is $100 \%$ at minimal one year follow up and at median 3 years follow up. One recipient developed dual kidney graft failure 10 years post-transplant secondary to acute rejection and also the patient was reported to be non-compliant. Remuzzi et al. [9] have reported an excellent long-term survival of either single or dual kidney grafts from ECD donors provided that the kidneys underwent a histologic evaluation before transplantation.

The most concern of surgical complication is vascular complication in dual kidney transplant. AI-Mamari et al. [10] reported $13 \%$ of renal vessel thrombosis of second kidney graft in 15 cases of unilateral dual kidney transplantation and resulted in graft loss. Hobart et al. [9,11] encountered $21 \%$ of vessel thrombosis and stenosis in 33 paediatric donors en bloc kidney transplant to the adults. There is no vascular complication in our series. Care must be taken to avoid the vessel kink during vessel anastomosis. The kidney graft position must be checked to ensure no vessel kinked before the wound closure. The upper pole of inferior kidney was usually parallel medially with lower pole of superior kidney graft. The two ureters were paralleled joining at the end forming one anastomosis to the bladder. There are two recipients who developed lymphocele in this series. One of them who had a midline incision with bilateral placement of kidney graft subsequently developed the ureteric stricture on the left side. Another case was unilateral placement of dual kidney grafts, in which dual ureters developed ureteric stenosis. Both cases recovered satisfactory after laparoscopic lymphocele fenestration and surgical reconstruction of urinary tract. It seems that the ureteric stricture is associated with lymphocele formation in our cohort. There was no urine leakage occurred in this series. In single kidney transplant, the incidence of urological complications was reported from 3.6 to $5.8 \%$ [12-14].

There were two patients who had delayed graft function (DGF) in this series. One of them was UDKT and the other was EDKT. In both cases, a prolonged cold ischaemic time (CIT) was noted at
726 minutes and 960 minutes. One of the donors had high SCr (165 $\mu \mathrm{mol} / \mathrm{L}$ ) at the time of kidney procument. The incidence of DGF has been reported from $20 \%$ to $30 \%$ in dual kidney transplant $[12,15]$. The incidence of DGF may be attributed to longer CIT $[16,17]$. The CIT and DGF are the most important predictors for short and long-term graft survival. The efforts should be made to minimize CIT under any circumstance.

The technique for dual kidney transplant has been evolved over last two decades. The dual kidney transplants can be performed as a single kidney transplant on both sides of the iliac fossa. Alternatively, the midline incision can be used for placing one graft in each side of iliac fossa. The disadvantage of these two techniques is that both sides of iliac fossa are utilized. Recently, unilateral placement of dual kidney grafts has been explored with advantages of spare of one side iliac fossa, time saving and equivalent graft outcome [18]. One of DKT in this series is his third time of kidney transplant. The dual grafts were placed at right iliac fossa successfully. The patient recovered well without any surgical complication. In addition, En bloc transplant at unilateral side is in favour using aorta and vena cava for vessel anastomosis [19].

In conclusion, DKT is an option for kidney transplantation from ECD. The excellent outcome can be achieved. Care needs to be taken during vessel anastomosis and position of dual kidney grafts to prevent vascular complication and graft loss. The CIT should be minimized to reduce DGF and improve long-term graft survival.

\section{References}

1. Mallon DH, Summers DM, Bradley JA, Pettigrew GJ (2013) Defining delayed graft function after renal transplantation: simplest is best. Transplantation 96: 885-889.

2. Kayler L, Zendejas I, Molmenti E, Chordia P, Schain D, et al. (2012) Kidney transplant ureteroneocystostomy: comparison of full-thickness vs. LichGregoir techniques. Clinical transplantation 26: E372-380.

3. Pascual J, Zamora J, Pirsch JD (2008) A systematic review of kidney transplantation from expanded criteria donors. American journal of kidney diseases : the official journal of the National Kidney Foundation 52: 553-586.

4. Johnson LB, Kno PC, Dafoe DC, Schweitzer EJ, Alfrey EJ, et al. (1996) Double adult renal allografts: a technique for expansion of the cadaveric kidney donor pool. Surgery 120: 580-583.

5. Moore PS, Farney AC, Sundberg AK, Rohr MS, Hartmann EL, et al. (2006) Experience with dual kidney transplants from donors at the extremes of age. Surgery 140: 597-605.

6. Laftavi MR, Stephan R, Feng L, Said M, Kohli R, et al. (2011) Dual kidney transplants from very old or very young donors: long-term outcomes and complications. Transplantation proceedings 43: 507-511.

7. Lu AD, Carter JT, Weinstein RJ, Prapong W, Salvatierra O, et al. (1999) Excellent outcome in recipients of dual kidney transplants: a report of the first 50 dual kidney transplants at Stanford University. Archives of surgery.134: 971-975.

8. Medina-Polo J, Pamplona-Casamayor M, Miranda-Utrera N, Gonzalez-Monte E, Passas-Martinez JB, et al. (2014) Dual kidney transplantation involving organs from expanded criteria donors: a review of our series and an update on current indications. Transplantation proceedings 46: 3412-3415.

9. Remuzzi G, Cravedi P, Perna A, Dimitrov BD, Turturro M, et al. (2006) Long term outcome of renal transplantation from older donors. The New Eng J Med 354: 343-352.

10. Hobart MG, Modlin CS, Kapoor A, Boparai N, Mastroianni B, et al. (1998) 
Transplantation of pediatric en bloc cadaver kidneys into adult recipients. Transplantation 66: 1689-1694.

11. Dalgic A, Boyvat F, Karakayali H, Moray G, Emiroglu R, et al. (2006) Urologic complications in 1523 renal transplantations: The Baskent University experience. Transplantation proceedings 38: 543-547.

12. Faenza A, Nardo B, Catena F, Scolari MP, d'Arcangelo GL, et al. (1999) Ureteral stenosis after kidney transplantation. A study on 869 consecutive transplants. Transpl Int 12: 334-340.

13. Faenza A, Nardo B, Fuga G, Liviano-D’Arcangelo G, Grammatico F, et al. (2005) Urological complications in kidney transplantation: ureterocystostomy versus uretero-ureterostomy. Transplantation proceedings 37: 2518-2520.

14. Gill J, Cho YW, Danovitch GM, Wilkinson A, Lipshutz G, et al. (2008) Outcomes of dual adult kidney transplants in the United States: an analysis of the OPTN/UNOS database. Transplantation 85: 62-68.
15. Lucarelli G, Bettocchi C, Battaglia M, Impedovo SV, Vavallo A, et al. (2010) Extended criteria donor kidney transplantation: comparative outcome analysis between single versus double kidney transplantation at 5 years. Transplantation proceedings 42: 1104-1107.

16. Rigotti P, Capovilla G, Di Bella C, Silvestre C, Donato P, et al. (2014) A single-center experience with 200 dual kidney transplantations. Clinical transplantation 28: 1433-1440.

17. Kayler LK, Srinivas TR, Schold JD (2011) Influence of CIT-induced DGF on kidney transplant outcomes. Am J Transplant 11: 2657-2664.

18. Quiroga I, McShane P, Koo DD, Gray D, Friend PJ, et al. (2006) Major effects of delayed graft function and cold ischaemia time on renal allograft survival. Nephrol Dial Transplant 21: 1689-1696.

19. Ekser B, Furian L, Broggiato A, Silvestre C, Pierobon ES, et al. (2010) Technical aspects of unilateral dual kidney transplantation from expanded criteria donors: experience of 100 patients. Am J Transplant 10: 2000-2007. 